\title{
When overlap leads to competition: Effects of phonological encoding on word duration
}

\author{
Loretta K. Yiu $^{1}$ - Duane G. Watson ${ }^{1}$
}

Published online: 9 April 2015

(C) Psychonomic Society, Inc. 2015

\begin{abstract}
Some accounts of acoustic reduction propose that variation in word duration is a reflection of the speaker's internal production processes, but it is unclear why lengthening within a word benefits planning. The present study examines whether variability in word length is partly attributable to phonological encoding. In an event-description task, speakers produced words with longer durations when the word shared part of its phonology with a previously articulated word than when it did not. More importantly, lengthening was greater when the overlap was word-initial than when it was wordfinal. These differences in duration are in line with predictions of serial phonological competition models, which claim that words that overlap in onsets create more competition than words that overlap in offsets and are thus more difficult to produce. That word duration is sensitive to differences in production difficulty suggests a link between speakers' duration choices and phonological encoding. We propose that lengthening provides the production system with the necessary processing time to produce a word's sounds.
\end{abstract}

Keywords Acoustic reduction · Word duration $\cdot$ Processing time

It is well established that speakers lengthen or shorten their utterances depending on context. Words that are new, infrequent, or less predictable tend to be acoustically prominent

Duane G. Watson

dgwatson@illinois.edu

1 Department of Psychology, University of Illinois at UrbanaChampaign, 603 E Daniel St., Champaign, IL 61820, USA whereas repeated, frequent, or predictable words are generally shorter and less intelligible (Aylett \& Turk, 2004; Bell, Brenier, Gregory, Girand, \& Jurafsky, 2009; Fowler \& Housum, 1987; Jurafsky, Bell, Gregory, \& Raymond, 2001; Lam \& Watson, 2010; inter alia). Why such attenuation occurs is still under debate, but explanations can be broadly grouped into two accounts. Communicative accounts suggest that speakers tailor their speech to optimize the acoustic signal for the listener by increasing the intelligibility of words that might otherwise be difficult to comprehend (Aylett \& Turk, 2004; Jaeger, 2010). In contrast, production ease accounts argue that repetition reduction is the product of the speaker's internal production system and represents what is easiest for the speakers themselves rather than the listeners' informational needs (Bell et al., 2009; Kahn \& Arnold, 2015). Although these two accounts are not incompatible (Galati \& Brennan, 2010; Jaeger, 2013), the present study will focus on a prediction specific to the latter account. The goal of this study is to examine whether or not duration differences are partly a reflection of the production processes involved in phonological encoding.

The speech production system is often thought to be characterized by a multistep process during which lexical information is accessed and then individual phonemes associated with that lemma are retrieved (e.g., Levelt, Roelofs, \& Meyer, 1999). The phonological representation in turn serves as input for the articulatory system. Production ease accounts argue that the degree of duration reduction on a word is related to the speed and ease of lexical access and retrieval (e.g., Bell et al., 2009; Kahn \& Arnold, 2015; Lam \& Watson, 2010). Words that are retrieved more quickly are produced with shorter durations. Words that have been previously mentioned may be retrieved more easily because representations at both the lexical and phonological levels are likely to be primed. In contrast, words that are new to the discourse may require more 
effort to plan because their relative activation levels are lower. Consequently, repeated words will be more reduced than when they are initially mentioned.

However, this work raises a question: Why might lengthening be linked to planning difficulty? Presumably, once articulation has begun, the word's meaning and lemma have already been accessed, so how could lengthening benefit the production system? One possibility is that the lengthening of a word specifically benefits phonological encoding processes that are still ongoing as the word is produced. Here we define phonological encoding as the processes engaged in selecting and ordering a word's sounds during production. Evidence from the literature suggests that phonological priming may enjoy a special status in repetition reduction. Kahn and Arnold (2012) observed that both mentioned and non-mentioned, but conceptually given, words exhibit reduction, but the amount of reduction was greater when speakers heard the repeated item spoken aloud. In a later study, they found that prior articulation of a word led to more reduction than merely thinking about it (Kahn \& Arnold, 2015). Furthermore, Lam and Watson (2014) found that repeated words led to reduction whereas repeated mention of referents did not. These studies suggest that duration variation may originate at the phonological level.

Traditional phonological competition models (e.g., O'Seaghdha, Dell, Peterson, \& Juliano, 1992) include nodes for words, phonemes, and connections between them. Activation of a target word spreads downward to its component sounds. These phonemes in turn activate nodes for other words that share sounds with the target, thus creating competition between discrepant phonemes to occupy slots in the phonological frame, which specifies the order of the word's sounds. Although these models assume that all phonemes of the target word are activated simultaneously, others have argued that phonological selection is a serial process (Sevald \& Dell, 1994; but see O'Seaghdha \& Marin, 2000). When a target word is activated, its component sounds are accessed in the order in which they appear in the word, starting with phonemes at the beginning of the word. Sevald and Dell (1994) found that rapid repetition of sequences of alternating words was faster when the words overlapped finally (e.g., PICK-TICK) than when they overlapped initially (e.g., PICK-PIN), and the size of the effect depended on the degree of overlap between the two words. They explain this effect through serial activation of the phonemes. If activation spreads serially, competition will occur between sounds that follow those that overlap. Thus, onset overlap will engender a longer period of lexical competition than final overlap, resulting in the miscuing of subsequent phonemes and delaying the correct selection of the target. In contrast, final overlap will result in less competition because by the time the shared sounds produce competition, nothing remains in the word to be miscued.
If phonological encoding is a serial process, as the evidence above suggests, then lengthening the word might provide speakers with more planning time. Thus, investigating the effects of phonological overlap on duration may provide clues as to whether repetition reduction is linked to phonological encoding. Previous work, however, has generally examined the speed and accuracy of producing a word following the visual presentation or articulation of a phonologically related word (the prime) rather than the duration of the word itself (e.g., Jescheniak, Schriefers, \& Hantsch, 2003; O'Seaghdha \& Marin, 2000). Even so, there do seem to be differences in production difficulty depending on the site of overlap, though the asymmetry has been inconsistent. When the target word overlaps in onset with what was previously articulated, production tends to be delayed (Janssen \& Caramazza, 2009, Experiment 2; Wheeldon, 2003) or is marked by more disfluencies (Jaeger, Furth, \& Hilliard, 2012b). In contrast, when the target word overlaps finally, production is generally facilitated compared to onset-related targets (O'Seaghdha \& Marin, 2000; Sullivan \& Riffel, 1999) or to phonologically unrelated targets (Sevald \& Dell, 1994). In addition, lexical selection is biased against words that overlap in onset (Jaeger, Furth, \& Hilliard, 2012a), but speakers are more likely to complete a fragment with a target word when the fragment contains a rhyme prime (Rapp \& Samuel, 2002). Others, however, have actually found facilitation in naming latencies for onset-related targets (Carreiras, Ferrand, Grainger, \& Perea, 2005; Janssen \& Caramazza, 2009, Experiment 1a; Meyer, 1990) but not targets that overlap finally, though the benefit seems to disappear when the sounds are the same but are spelled differently (Damian \& Bowers, 2003) or when the prime and target are part of different phrases (Smith \& Wheeldon, 2004).

The few studies that have looked at the effects of phonological overlap on durations of single- and multi-word utterances have yielded mixed results as well. Some have found that word durations remain unaffected in phonologically similar contexts even when speech onsets differ (e.g., Damian, 2003; Damian \& Dumay, 2009; Meyer, 1990), while others have observed a slow-down in speech rate when producing words that overlap initially (Jaeger et al., 2012b; Sevald \& Dell, 1994). More recently, a simple recurrent network designed to simulate a serial phonological encoding network accurately predicted that overlap could lead speakers to lengthen segments within a word. Watson, Buxó-Lugo, and Simmons (in press) trained a model to produce pairs of words in succession that either overlapped in their initial morpheme (e.g., LAYOUT-LAYOVER) or in their final morpheme (e.g., OUTLAY-OVERLAY). The network experienced the most errors when producing words that overlapped initially, replicating the pattern from Sevald and Dell (1994). It also produced more errors when producing the non-overlapping part of the word. When speakers had to produce alternating word 
sequences similar to those the model produced, they slowed down during the morpheme that did not overlap, the same region where the model predicted the most production difficulty. Thus, the parts of the word that were the most difficult to produce seemed to correspond with lengthening, suggesting that phonological selection is a serial process. If it is serial, lengthening during articulation could provide more time for the production system to select the correct phonemes at points at which competition occurs. It is unclear, however, whether the interference elicited by these word repetition tasks taps the same processes that are engaged in word production in a connected discourse. It is possible that repeating two words rapidly in succession over and over again may create competition and lengthening that might not occur in natural connected conversation. Thus, to show that reduction in discourse is partly a product of production factors, one would need to demonstrate these effects in a more natural context.

In the present study, we examined whether the phonological overlap effects on duration that are predicted by a serial phonological encoding model of production generalize to reduction and lengthening in discourse structure. In an event description task, we manipulated the location of phonological overlap with a previously articulated word to determine whether the type of overlap affects the duration of a target word in a sentence. If lengthening is linked to difficulty in phonological planning, then target words that overlap initially with a prime should exhibit more lengthening than target words that overlap finally due to competition early in the phonological selection process, a burden not experienced in cases of final overlap. However, if word duration is not sensitive to the presence or location of phonological competition, then we should not expect any duration differences between initial and final overlap.

\section{Methods}

\section{Participants}

Fifty-two individuals participated in this study in exchange for course credit or compensation of $\$ 8$. All participants were native English speakers and reported normal or corrected to normal vision and hearing.

\section{Materials and design}

The participants' task was to describe events on a computer screen. Participants were presented with four images arranged in a $2 \times 2$ display on each trial. During each trial, one object would shrink (the prime), and then one object (the target) would flash.
A total of 320 images were used. A subset of the images was taken from the colorized versions of the Snodgrass and Vanderwart (1980) dataset (Rossion \& Pourtois, 2001). The 32 critical trials were made up of 128 images grouped such that each critical trial contained a target item, an object whose name overlapped initially with the target, an object whose name overlapped finally with the target, and an object whose name did not share any phonological overlap with the target. The critical trials were divided into four conditions, with eight trials in each. On the initial overlap and final overlap trials, the target object overlapped initially or finally in phonology with its prime, respectively. Given and new trials were included as baseline comparisons. On given trials, the target object was a repeat of its prime. On new trials, there was no phonological overlap between the target and the prime. Examples of utterances from each of the conditions are given below:

a) Initial overlap: The beetle shrinks. The beaker flashes.

b) Final overlap: The speaker shrinks. The beaker flashes.

c) Given: The beaker shrinks. The beaker flashes.

d) New: The apple shrinks. The beaker flashes.

The list of critical items can be found in Appendix Table 4. The critical items were counterbalanced using a Latin square, resulting in four lists. This allowed us to compare the production of the same target across conditions. The remaining images made up the 48 filler trials in which the shrinking object did not share any phonological overlap with the flashing object. The locations of the shrinking and flashing objects were randomized. The order of the trials was pseudo-randomly permuted for each participant such that no trials involving any phonological overlap would appear in succession.

\section{Procedure}

To maximize the correct naming of the target objects, participants were familiarized with the stimuli prior to the experiment. During this familiarization phase, participants viewed each image individually with its corresponding name printed to the side. Participants were encouraged to use the names they saw in this phase to describe the shrinking and flashing objects in the experiment.

The experiment began with three practice trials. One of the targets was a repeated mention and the other two were nonrepeated mentions. The images in the practice trials were not used in the experimental trials. At the beginning of a trial, four images appeared on the screen. After $2 \mathrm{~s}$, the prime image would shrink. Then, after $1 \mathrm{~s}$, the target image would flash. Participants were told to describe each event as soon as they knew what was happening. All trials were recorded with a microphone headset. 


\section{Results}

Target word boundaries were marked by the first author and durations were extracted using the speech analysis platform Praat (Boersma \& Weenink, 2007). To ensure the validity of the coding, a second coder naïve to the experimental conditions and predictions also labeled the conditions. There was no difference in effects between the two coding sets, so the first author's coding is reported below. Approximately $3.7 \%$ of the trials were discarded because the target was either misnamed or produced with a disfluency. The number of discarded trials did not differ across conditions. Duration data are presented in Fig. 1.

Results were analyzed using a linear mixed effects model with condition as a fixed effect and a maximal random effects structure. Durations were log-transformed and scaled. Condition was coded using Helmert contrasts and centered. Helmert coding compares each level of a factor with the average of its subsequent levels. Tables 1 and 2 display parameter estimates for the model.

The first Helmert contrast compared the mean duration of target words in the given condition ( $418 \pm 6 \mathrm{~ms}$ ) to the mean duration in the other three conditions where the target word was not a repeat of its prime $(467 \pm 7 \mathrm{~ms})$. As expected, repeated lexical items had shorter durations than nonrepeated lexical items $(\beta=.33, S E=.05, t=6.79, p<.001)$.

The second Helmert contrast then compared the mean duration of target words in the new condition $(445 \pm 6 \mathrm{~ms})$ to the mean duration in the two conditions in which the target was a new item but shared some phonology with its prime $(479 \pm 7 \mathrm{~ms})$. Partial phonological overlap led to longer durations than when there was no overlap between the target and the prime $(\beta=.24, S E=.04, t=5.81, p<.001)$.

The third Helmert contrast was of most interest for our theoretical question. It examined whether there were duration differences depending on the site of phonological overlap (initial vs. final). Target words that overlapped initially with their

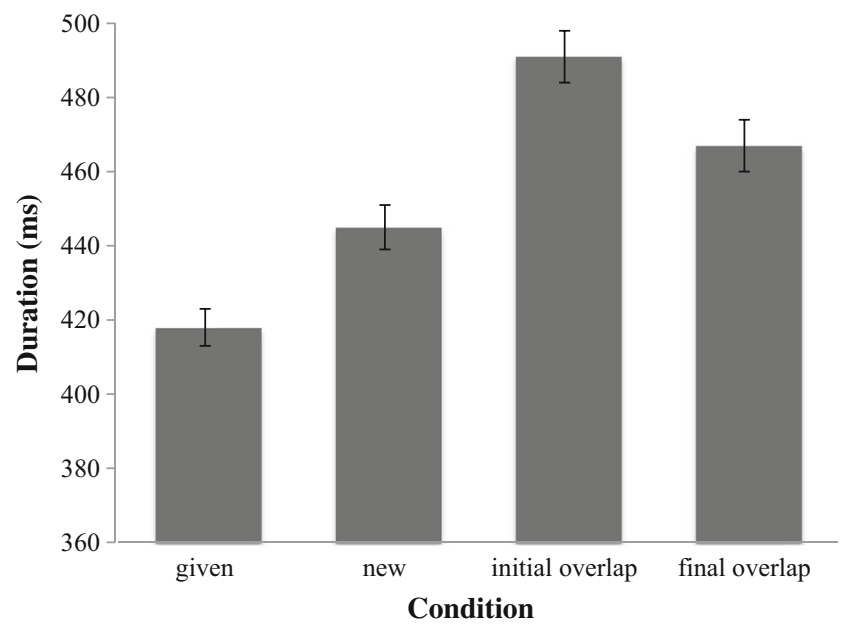

Fig. 1 Target word durations for each condition in milliseconds. Error bars indicate standard error
Table 1 Fixed effects estimates for target word durations

\begin{tabular}{lrrr}
\hline Fixed effects & Estimate & $\begin{array}{l}\text { Standard } \\
\text { error }\end{array}$ & t-value \\
\hline (Intercept) & -0.003 & 0.128 & -0.023 \\
$\begin{array}{l}\text { Given vs. new/initial overlap/final } \\
\quad 0 v e r l a p\end{array}$ & 0.328 & 0.048 & 6.794 \\
New vs. initial overlap/final overlap & 0.240 & 0.041 & 5.813 \\
Initial overlap vs. final overlap & -0.159 & 0.052 & -3.075 \\
\hline
\end{tabular}

primes were produced with longer durations $(491 \pm 7 \mathrm{~ms})$ than words that overlapped finally $(467 \pm 7 \mathrm{~ms})(\beta=-.16$, $S E=.05, t=-3.08, p=.002$ ).

Since there is a potential for contrast when producing two similar sounding words in succession, items in the two overlap conditions may have been produced with contrastive accents. Given that contrastively focused elements are generally produced with increased durations (e.g., Breen, Fedorenko, Wagner, \& Gibson, 2010; Cooper, Eady, \& Mueller, 1985), it is possible that the observed duration differences between the initial and final overlap conditions is due not to increased competition in the initial overlap condition, but rather due to differences in the degree of acoustic prominence.

To address this concern, we conducted an analysis of several measures of F0. In English, pitch accents that are associated with contrast typically have larger F0 excursions than non-contrastive pitch accents (Ladd, 2008; Pierrehumbert \& Hirschberg, 1990). Thus, if speakers are more likely to produce a contrastive accent for items in the initial overlap condition, the average F0 maximum height should be larger in the initial overlap condition than in the final overlap and new conditions. Similarly, if speakers accent both syllables more often in the initial overlap condition than in the final overlap or new conditions, then the average F0 should be higher across the word in the initial overlap condition. Table 3 lists the relevant F0 values for each condition.

There were no differences between the new and overlap conditions for either mean $\mathrm{F} 0$ values $(\beta=2.24, S E=2.06, t=1.09$, $p=.28)$ or maximum F0 values $(\beta=-5.96, S E=6.79$, $t=-0.88, p=.38$ ). Furthermore, there were no differences between the initial overlap and final overlap conditions for either mean F0 ( $\beta=-0.29, S E=2.88, t=-0.10, p=.92)$ or maximum F0 values $(\beta=-1.38, S E=7.61, t=-0.18, p=.86)$. The only reliable difference was between the given condition and the other conditions $(p<0.01)$, suggesting that although speakers were accenting focused words as one might expect, they did not appear to do so differently within the focused conditions.

\section{Discussion}

The present study examined whether speakers' durational choices may partly be attributable to the process of phonological 
Table 2 Random effects estimates for target word durations

\begin{tabular}{llll}
\hline Random effects & Name & Variance & Standard deviation \\
\hline Subject & (Intercept) & 0.354 & 0.595 \\
Subject & Given vs. new/initial overlap/final overlap & 0.045 & 0.213 \\
Subject & New vs. initial overlap/final overlap & 0.021 & 0.144 \\
Subject & Initial overlap vs. final overlap & 0.024 & 0.156 \\
Item & (Intercept) & 0.297 & 0.545 \\
Item & Given vs. new/initial overlap/final overlap & 0.013 & 0.113 \\
Item & New vs. initial overlap/final overlap & 0.005 & 0.070 \\
Item & Initial overlap vs. final overlap & 0.021 & 0.144 \\
\hline
\end{tabular}

encoding. When speakers produced words that partially overlapped in phonology with a previously articulated word in our event description task, they lengthened the target words to a greater extent when the words overlapped initially with their primes than when they overlapped finally. This difference is in line with predictions of serial phonological competition models that propose that words that overlap initially are more difficult to produce than words that overlap finally because discrepant segments are selected after the shared ones rather than before (Sevald \& Dell, 1994). That lengthening seems to correspond with increased levels of phonological competition suggests that speakers' durational choices are linked to difficulty in phonological planning.

Although a fully parallel model would not predict duration differences between the initial and final overlap conditions, phonological encoding may not be strictly serial either. Partial phonological overlap seems to be generally inhibitory, for target words that partially overlapped with their primes were produced with longer durations than words that did not share any phonological overlap at all. This is in contrast to Sevald and Dell's (1994) finding that the production of pairs with final overlap was slightly facilitated compared to unrelated controls. Instead, our findings may be better explained by O'Seaghdha and Marin's (2000) parallel-then-sequential phonological competition model. Their model proposes that all segments are first activated, thus providing locationindependent feedback between form-related words. In other words, any similarity between the target and the prime without repeating the whole word would increase the chance for contrasting segments between the two to move from the phonological plan of one word to the other. The activated segments are then assigned sequentially, further increasing the competition for words that overlap initially but not those that overlap finally. Lengthening may have occurred for both overlap conditions to provide the production system with more time to select the correct phonemes, with longer durations in the initial overlap condition providing the system with even more time to resolve the increased competition during the serial assignment process. In short, duration choices may allow time for phonological selection without assuming a strictly serial architecture.

There are two possibilities for how lengthening is realized within a word. One possibility is that the system is incremental. Speakers slow down either before or during points within the word at which interference is maximal. In the initial overlap case, for example, this would predict that lengthening occurs either before or during the phoneme that differs. The second possibility is that the system slows down where it can. In order to cope with interference during phonological encoding, lengthening occurs at locations where lengthening can be maximized, such as on vowels, the result being that lengthening is independent of the location of maximal interference. Future work will need to be done to test which of these two phoneme level predictions are correct, and such testing may potentially shed light on production mechanisms more generally. It is worth emphasizing, however, that independent of which of the aforementioned theories is correct, the work here confirms the production-centered prediction that there is greater lengthening in the initial overlap condition than in the final overlap condition.

It is important to note that phonological encoding is not the only factor that affects word duration. Speech rate, predictability, frequency, and other communicative factors may map onto speakers' durational choices as well (e.g., Jurafsky et al., 2001; Lam \& Watson, 2010, and many others). One potential

Table 3 F0 values for each condition. Standard errors are given in parentheses

\begin{tabular}{lllll}
\hline & Given & New & Initial overlap & Final overlap \\
\hline Average F0 (Hz) & $163.29(2.53)$ & $165.63(2.44)$ & $168.59(2.58)$ & $168.77(2.73)$ \\
Maximum F0 (Hz) & $214.67(5.32)$ & $233.54(5.84)$ & $227.63(5.38)$ & $227.88(5.59)$ \\
Minimum F0 (Hz) & $140.34(2.44)$ & $135.60(2.40)$ & $136.72(2.57)$ & $139.09(2.58)$ \\
\hline
\end{tabular}


listener-centered explanation is that speakers lengthen the confusable part of the words in order to optimize comprehension for the listener. There is a great deal of evidence, primarily from researchers who have used information theoretic approaches to understanding language, suggesting that this is true (e.g., Aylett \& Turk, 2004). In the current experiment, such an approach would predict that speakers would lengthen the parts of the target word that were most informative (i.e., the end of the words for initial overlap and the beginning of the words for final overlap). Critically, however, although communicative factors undoubtedly have an effect on word duration, it is less clear how they would account for the lengthening observed in the initial overlap relative to the final overlap condition. In contrast, this is a straightforward prediction of the production account. Initial overlap leads to early interference that lasts throughout the word, while final overlap leads to interference that comes in relatively late. Furthermore, the current experiment was designed to deemphasize any communicative factors that might be playing a role. Given that the same target words were produced across conditions, factors such as entropy or word frequency should be balanced across all conditions and therefore have little influence on speaker duration. In addition, there was no overt listener, participants experienced a large number of trials in which target phonological overlap occurred, and descriptions of the pictures were fairly rote. Thus, although it is certainly possible that speakers differentially lengthen words that overlap initially and words that overlap finally in other contexts, the current experiment was designed to minimize this possibility.

Serial phonological encoding entails that phonological selection varies in encoding difficulty over time, but how this serial assignment process interacts with other factors, such as phonological neighborhood density, is open to further investigation. In general, words with dense neighborhoods are more quickly and accurately named than words with sparse neighborhoods (e.g., Vitevitch, 2002), but the presence of phonologically similar neighbors tends to lead to hyperarticulation within the word (Baese-Berk \& Goldrick, 2009; Scarborough, 2012; but see Gahl, Yao, \& Johnson, 2012). If the effects of both phonological neighborhood density and phonological overlap arise from similar sources, then the location within the word where neighbors can be formed may be predictive of planning difficulty. Having a high proportion of neighbors sharing the onset of a word may hinder the encoding process because the intended phonological segments following the shared initial portion would face increased competition from the many other segments activated by the neighboring word forms, slowing articulation. In line with this prediction, Goldrick, Vaughn, and Murphy (2013) found that minimal pair neighbors enhance the voicing contrast in initial positions, but not in word-final positions. Dense onsets have also been found to slow naming latencies when overall neighborhood density, phonotactic probability, and word frequency are controlled for (Vitevitch, Armbrüster, \& Chu, 2004). These findings suggest that phonological overlap can interact with other factors that contribute to difficulty in lexical planning and articulation, and future work exploring these relationships may help further specify the mechanisms underlying word lengthening.

In sum, the present study suggests that the production system needs more time to produce a word when its phonological plan has overlapping segments with a previously articulated word, especially when the overlap is word-initial. The finding that duration is sensitive to the presence and location of phonological overlap points toward a link between word duration and planning difficulty, a relationship that listeners could potentially exploit in processing. Lengthening a word may benefit its own articulation by providing the production system with the necessary time to produce the word's sounds.

Acknowledgments This project was supported by Grant Number R01DC008774 from the National Institute on Deafness and Other Communication Disorders and a grant from the James S. McDonnell Foundation. We thank Florian Jaeger for helpful comments on an earlier version of this manuscript.

\section{Appendix}

Table 4 List of critical stimuli

\begin{tabular}{lll}
\hline Target word & Initial overlap & Final overlap \\
\hline bat & bag & hat \\
beach & beak & peach \\
beaker & beetle & speaker \\
bell & belt & shell \\
brick & bridge & stick \\
candle & candy & sandal \\
carrot & carriage & parrot \\
castle & casket & tassel \\
chair & chain & bear \\
clock & claw & sock \\
clown & cloud & crown \\
cradle & crater & ladle \\
dollar & dolphin & collar \\
ferry & ferret & cherry \\
goat & ghost & boat \\
heart & harp & dart \\
horn & horse & corn \\
knight & knife & kite \\
lamb & lamp & jam \\
letter & lettuce & sweater \\
mask & map & flask \\
moth & mop & sloth
\end{tabular}


Table 4 (continued)

\begin{tabular}{lll}
\hline Target word & Initial overlap & Final overlap \\
\hline mouse & mouth & house \\
pickle & picture & nickel \\
pin & pig & fin \\
plane & plate & rain \\
racket & rabbit & jacket \\
rose & rope & hose \\
saddle & salmon & paddle \\
snail & snake & whale \\
spoon & spool & moon \\
suit & soup & boot \\
\hline
\end{tabular}

\section{References}

Aylett, M., \& Turk, A. (2004). The smooth signal redundancy hypothesis: A functional explanation for relationships between redundancy, prosodic prominence, and duration in spontaneous speech. Language and Speech, 47, 31-56.

Baese-Berk, M., \& Goldrick, M. (2009). Mechanisms of interaction in speech production. Language and Cognitive Processes, 24, 527-554.

Bell, A., Brenier, J., Gregory, M., Girand, C., \& Jurafsky, D. (2009). Predictability effects on durations of content and function words in conversational English. Journal of Memory and Language, $60,92-111$.

Boersma, P., \& Weenink, D. (2007). Praat: Doing phonetics by computer (Version 4.5.14) [Computer software]. Retrieved from http://www. praat.org/

Breen, M., Fedorenko, E., Wagner, M., \& Gibson, E. (2010). Acoustic correlates of information structure. Language and Cognitive Processes, 25, 1044-1098.

Carreiras, M., Ferrand, L., Grainger, J., \& Perea, M. (2005). Sequential effects of phonological priming in visual word recognition. Psychological Science, 16, 585-589.

Cooper, W. E., Eady, S. J., \& Mueller, P. R. (1985). Acoustical aspects of contrastive stress in question-answer contexts. The Journal of the Acoustical Society of America, 77, 2142-2156.

Damian, M. F. (2003). Articulatory duration in single word speech production. Journal of Experimental Psychology: Learning, Memory, and Cognition, 29, 416-431.

Damian, M. F., \& Bowers, J. S. (2003). Effects of orthography on speech production in a form-preparation paradigm. Journal of Memory and Language, 49, 119-132.

Damian, M. F., \& Dumay, N. (2009). Exploring phonological encoding through repeated segments. Language and Cognitive Processes, 24, 685-712.

Fowler, C. A., \& Housum, J. (1987). Talkers' signaling of "new" and "old" words in speech and listeners' perception and use of the distinction. Journal of Memory and Language, 26, 489-504.

Gahl, S., Yao, Y., \& Johnson, K. (2012). Why reduce? Phonological neighborhood density and phonetic reduction in spontaneous speech. Journal of Memory and Language, 66, 789-806.

Galati, A., \& Brennan, S. E. (2010). Attenuating information in spoken communication: For the speaker, or for the addressee? Journal of Memory and Language, 62, 35-51.
Goldrick, M., Vaughn, C., \& Murphy, A. (2013). The effects of lexical neighbors on stop consonant articulation. The Journal of the Acoustical Society of America, 134, EL172-EL177.

Jaeger, T. F. (2010). Redundancy and reduction: Speakers manage syntactic information density. Cognitive Psychology, 61, 23-62.

Jaeger, T. F. (2013). Production preferences cannot be understood without reference to communication. Frontiers in Psychology, 4, 230.

Jaeger, T. F., Furth, K., \& Hilliard, C. (2012a). Phonological overlap affects lexical selection during sentence production. Journal of Experimental Psychology: Learning, Memory, and Cognition, 38, $1439-1449$.

Jaeger, T. F., Furth, K., \& Hilliard, C. (2012b). Incremental phonological encoding during unscripted sentence production. Frontiers in Psychology, 3, 1-22.

Janssen, N., \& Caramazza, A. (2009). Grammatical and phonological influences on word order. Psychological Science, 20, 1262-1268.

Jescheniak, J. D., Schriefers, H., \& Hantsch, A. (2003). Utterance format affects phonological priming in the picture-word task: Implications for models of phonological encoding in speech production. Journal of Experimental Psychology: Human Perception and Performance, 29, 441-454.

Jurafsky, D., Bell, A., Gregory, M., \& Raymond, W. D. (2001). Probabilistic relations between words: Evidence from reduction in lexical production. In J. Bybee \& P. Hopper (Eds.), Frequency and the emergence of linguistic structure (pp. 229-254). Amsterdam: Benjamins.

Kahn, J. M., \& Arnold, J. E. (2012). A processing-centered look at the contribution of givenness to durational reduction. Journal of Memory and Language, 67, 311-325.

Kahn, J. M., \& Arnold, J. E. (2015). Articulatory and lexical repetition effects on durational reduction: Speaker experience vs. common ground. Language, Cognition, and Neuroscience, 30, 103-119.

Ladd, D. (2008). Intonational phonology (2nd ed.). Cambridge: Cambridge University Press.

Lam, T. Q., \& Watson, D. G. (2010). Repetition is easy: Why repeated references have reduced prominence. Memory \& Cognition, 38 , $1137-1146$.

Lam, T. Q., \& Watson, D. G. (2014). Repetition reduction: Lexical repetition in the absence of referent repetition. Journal of Experimental Psychology: Learning, Memory, and Cognition, 40, 829-843.

Levelt, W. J. M., Roelofs, A., \& Meyer, A. S. (1999). A theory of lexical access in speech production. Behavioral and Brain Sciences, 22, 1-38.

Meyer, A. S. (1990). The time course of phonological encoding in language production: The encoding of successive syllables of a word. Journal of Memory and Language, 29, 524-545.

O’Seaghdha, P. G., \& Marin, J. W. (2000). Phonological competition and cooperation in form-related priming: Sequential and nonsequential processes in word production. Journal of Experimental Psychology: Human Perception and Performance, 26, 57-73.

O'Seaghdha, P. G., Dell, G. S., Peterson, R. R., \& Juliano, C. (1992). Modeling form-related priming effects in comprehension and production. In R. Reilly \& N. E. Sharkey (Eds.), Connectionist approaches to language processing (Vol. 1). Hillsdale, N. J.: Erlbaum.

Pierrehumbert, J., \& Hirschberg, J. (1990). The meaning of intonational contours in the interpretation of discourse. In P. Cohen, J. Morgan, \& M. Pollack (Eds.), Intentions in communication (pp. 271-311). Cambridge, MA: MIT Press.

Rapp, D. N., \& Samuel, A. G. (2002). A reason to rhyme: Phonological and semantic influences on lexical access. Journal of Experimental Psychology: Learning, Memory, and Cognition, 28, 564-571.

Rossion, B., \& Pourtois, G. (2001). Revisiting Snodgrass and Vanderwart's object database: Color and texture improve object recognition. Journal of Vision, 1, 413a. 
Scarborough, R. (2012). Lexical similarity and speech production: Neighborhoods for nonwords. Lingua, 122, 164-176.

Sevald, C. A., \& Dell, G. S. (1994). The sequential cuing effect in speech production. Cognition, 53, 91-127.

Smith, M., \& Wheeldon, L. (2004). Horizontal information flow in spoken sentence production. Journal of Experimental Psychology: Learning, Memory, and Cognition, 30, 675-686.

Snodgrass, J. G., \& Vanderwart, M. (1980). A standardized set of 260 pictures: Norms for name agreement, image agreement, familiarity, and visual complexity. Journal of Experimental Psychology: Human Learning \& Memory, 6, 174-215.

Sullivan, M. P., \& Riffel, B. (1999). The nature of phonological encoding during spoken word retrieval. Language and Cognitive Processes, 14, 15-45.
Vitevitch, M. S. (2002). The influence of phonological similarity neighborhoods in speech production. Journal of Experimental Psychology: Learning, Memory, and Cognition, 28, 735-747.

Vitevitch, M. S., Armbrüster, J., \& Chu, S. (2004). Sublexical and lexical representations in speech production: Effects of phonotactic probability and onset density. Journal of Experimental Psychology: Learning, Memory, and Cognition, 30, 514-529.

Watson, D. G., Buxó-Lugo, A., \& Simmons, D. C. (in press). The effect of phonological encoding on word duration: Selection takes time. In E. Gibson \& L. Frazier (Eds.), Explicit and Implicit Prosody in Sentence Processing.

Wheeldon, L. (2003). Inhibitory form priming in spoken word production. Language and Cognitive Processes, 18, 81-109. 